\title{
Choroidal metastases and choroidal melanomas: comparison of ultrasonographic findings
}

\author{
Beate Sobottka, Torsten Schlote, Hans G Krumpaszky, Ingrid Kreissig
}

\begin{abstract}
Aims-The purpose of the study was to analyse, whether the shape and the height to base ratio in B-scan ultrasonography are appropriate to differentiate choroidal melanomas from metastases.

Methods-Between 1991 and 199616 eyes of 16 patients with choroidal metastases from breast carcinomas and 66 eyes of 66 patients with choroidal melanomas were evaluated ultrasonographically. The diagnosis of choroidal melanoma has been confirmed histologically in all eyes. Irradiated tumours were excluded from the study. Fisher's exact test and $\chi^{2}$ test were used for statistical analysis.

Results-Choroidal metastases demonstrated a significantly lower height to base ratio in $\mathrm{B}$-scan (mean $0.18, \mathrm{SD} 0.08$ ) than melanomas (mean $0.6(0.16) ; p<0.001)$. A polygonal tumour surface was significantly more frequent in metastases (13 out of 16 metastases and in six out of 66 melanomas, $p<0.001)$. A choroidal excavation could be demonstrated in 38 melanomas and in no metastatic tumour $(p<0.001)$. The reflectivity was significantly higher in metastases than in melanomas.

Conclusion-The combined use of height to base ratio and reflectivity enables a highly significant discrimination between choroidal melanomas and metastases from the breast, thus probably constituting appropriate variables for the clinical differentiation.
\end{abstract}

(Br F Ophthalmol 1998;82:159-161)

Ophthalmology III, University of Tuebingen, Germany B Sobottka

I Kreissig

Ophthalmology I, University of

Tuebingen, Germany

T Schlote

Department of Medical Information Processing, University of Tuebingen, Germany

H G Krumpaszky

Correspondence to: Beate Sobottka, MD, University Eye Clinic of Tuebingen,

Schleichstrasse 12, 72076

Tuebingen, Germany.

Accepted for publication 27 August 1997 graphically differentiated by evaluating refle tivity, internal structure, and degree of vascularisation. However, even the ultrasonographic picture of metastatic tumours (for example, metastasis of carcinoid tumour of the lung) may resemble choroidal melanomas. ${ }^{6}$

The purpose of this study was to analyse, whether variables of B-scan ultrasonography are appropriate to differentiate choroidal metastasis of the breast-as the most frequent metastatic tumour to the uvea-from choroidal melanoma.

\section{Methods}

Between 1991 and 199616 eyes of 16 female patients (mean age 60 (SD 15) years) with choroidal metastases from breast carcinomas and 66 eyes of 66 patients ( 35 female and 31 male patients; mean age 64 (13) years with choroidal melanomas were evaluated ultrasonographically, using a standardised A and B-mode system (Cooper Vision Digital B, Kretz 7200 MA, USA, Innovative Imaging I3). The diagnosis of choroidal melanoma has been confirmed histologically in all eyes after enucleation.

The A-mode transducer had the following specifications-nominal frequency of $8 \mathrm{MHz}$, non-focused, diameter $5 \mathrm{~mm}$; the B-mode transducer was of the type-mechanical sector scanning, frame rate 10 or $12 \mathrm{~Hz}$, nominal frequency of $10 \mathrm{MHz}$, focus at $20 \mathrm{~mm}$ from exit window of scan head. The transducer was applied posterior to the limbus and opposite the location of the tumour. The tumours were examined at $60 \mathrm{~dB}$ gain settings. B-mode variables were: base diameter, prominence, shape, choroidal excavation. A-mode variables were: prominence, reflectivity, regularity. The maximum height of the tumour obtained by A and B-mode was defined as prominence and the maximum dimension observed with B-mode as base diameter. For each tumour the height to base ratio was calculated. The tumours were classified as mushroom, dome, and polygonal shaped. A convex mass was defined as dome shaped when the growth of the lesion was restrained by Bruch's membrane. A mushroom-shaped surface was assumed when the tumour broke through Bruch's membrane. A polygonal tumour surface was defined as irregular surface contour without offering the picture of a mushroom-shaped lesion. Reflectivity quantified the mean echo amplitude of the tumour in percentage of the maximum display height. Irradiated tumours were excluded from the study. Data were analysed if significant differences of the ultrasonographic variables between choroidal melanomas and metastases of breast carcinoma existed. Fisher's exact test and $\chi^{2}$ test were used for statistical evaluation. 
Table 1 Echographic findings of choroidal metastases $(n=16)$

\begin{tabular}{llllrlll}
\hline No & HB ratio & Reflect & Shape & No & HB ratio & Reflect & Shape \\
\hline 1 & 0.17 & 60 & poly & 9 & 0.17 & 90 & poly \\
2 & 0.08 & 80 & poly & 10 & 0.09 & 90 & poly \\
3 & 0.15 & 60 & poly & 11 & 0.12 & 70 & poly \\
4 & 0.21 & 90 & poly & 12 & 0.21 & 80 & poly \\
5 & 0.26 & 80 & dome & 13 & 0.18 & 90 & poly \\
6 & 0.31 & 60 & dome & 14 & 0.12 & 80 & dome \\
7 & 0.40 & 70 & poly & 15 & 0.14 & 60 & poly \\
8 & 0.14 & 80 & poly & 16 & 0.16 & 70 & poly \\
\hline
\end{tabular}

${ }^{\star}$ Height to base ratio; †A-scan reflectivity in $\% ; \ddagger \mathrm{B}$-scan shape: dome $=$ dome shaped, mush $=$ mushroom shaped, poly = polygonal shaped.

Table 2 Echographic findings of choroidal melanomas $(n=66)$

\begin{tabular}{|c|c|c|c|c|c|c|c|}
\hline No & HB ratio * & Reflectt & Shape $\neq$ & No & HB ratio * & Reflectt & Shape $\neq$ \\
\hline 1 & 0.32 & 0 & dome & 34 & 0.86 & 20 & mush \\
\hline 2 & 0.58 & 30 & dome & 35 & 0.73 & 20 & dome \\
\hline 3 & 0.42 & 40 & dome & 36 & 0.83 & 50 & dome \\
\hline 4 & 0.52 & 20 & dome & 37 & 0.61 & 20 & dome \\
\hline 5 & 0.46 & 50 & dome & 38 & 0.85 & 20 & dome \\
\hline 6 & 0.48 & 30 & dome & 39 & 0.95 & 20 & mush \\
\hline 7 & 0.41 & 10 & poly & 40 & 0.66 & 10 & dome \\
\hline 8 & 0.34 & 10 & dome & 41 & 0.23 & 0 & dome \\
\hline 9 & 0.61 & 20 & much & 42 & 0.44 & 40 & dome \\
\hline 10 & 0.51 & 20 & dome & 43 & 0.57 & 40 & dome \\
\hline 11 & 0.61 & 40 & dome & 44 & 0.60 & 30 & dome \\
\hline 12 & 0.65 & 50 & mush & 45 & 0.40 & 0 & dome \\
\hline 13 & 0.53 & 20 & dome & 46 & 0.65 & 30 & dome \\
\hline 14 & 0.50 & 10 & dome & 47 & 0.75 & 10 & dome \\
\hline 15 & 0.63 & 10 & mush & 48 & 0.89 & 40 & mush \\
\hline 16 & 0.51 & 30 & dome & 49 & 0.75 & 10 & mush \\
\hline 17 & 0.41 & 0 & dome & 50 & 0.36 & 30 & dome \\
\hline 18 & 0.46 & 0 & dome & 51 & 0.46 & 0 & mush \\
\hline 19 & 0.74 & 40 & dome & 52 & 0.78 & 30 & mush \\
\hline 20 & 0.69 & 30 & dome & 53 & 0.82 & 0 & dome \\
\hline 21 & 0.62 & 10 & poly & 54 & 0.69 & 30 & mush \\
\hline 22 & 0.53 & 40 & dome & 55 & 0.64 & 10 & dome \\
\hline 23 & 0.63 & 40 & dome & 56 & 0.61 & 40 & dome \\
\hline 24 & 0.69 & 50 & mush & 57 & 0.60 & 0 & poly \\
\hline 25 & 0.46 & 0 & dome & 58 & 0.64 & 30 & dome \\
\hline 26 & 0.69 & 20 & dome & 59 & 0.55 & 0 & poly \\
\hline 27 & 0.84 & 40 & dome & 60 & 0.54 & 10 & poly \\
\hline 28 & 0.58 & 10 & dome & 61 & 0.71 & 10 & mush \\
\hline 29 & 0.51 & 20 & dome & 62 & 0.72 & 20 & dome \\
\hline 30 & 0.53 & 20 & mush & 63 & 0.83 & 50 & dome \\
\hline 31 & 0.76 & 20 & mush & 64 & 0.45 & 20 & dome \\
\hline 32 & 0.49 & 20 & dome & 65 & 0.68 & 30 & dome \\
\hline 33 & 0.36 & 0 & poly & 66 & 0.83 & 30 & dome \\
\hline
\end{tabular}

${ }^{\star}$ Height to base ratio; †A-scan reflectivity in $\%$; $\ddagger$ B-scan shape: dome $=$ dome shaped, mush $=$ mushroom shaped, poly $=$ polygonal shaped .

Diagnosis - Malignant melanoma o Ma-Ca metastasis

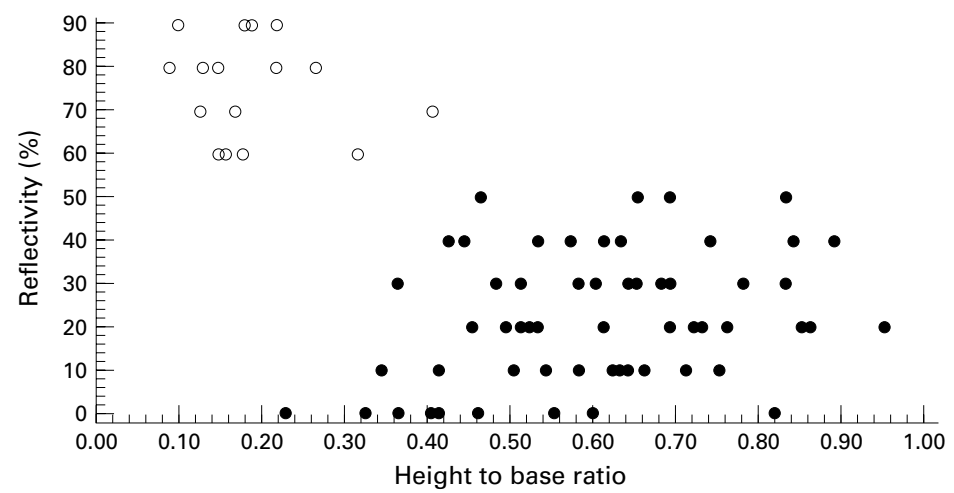

Figure 1 Comparison of metastases and melanomas concerning reflectivity (\%) and height to base ratio demonstrates a significant different distribution pattern.

Results

The results are summarised in Tables 1 and 2 .

TUMOUR DIMENSION

The prominence of the metastatic tumours (n $=16$ ) in B-scan ranged between 0.8 and $8 \mathrm{~mm}$ (mean 2.7 (SD 1.9) $\mathrm{mm}$ ). The base diameter of these tumours ranged between 4.3 and 16.3 $\mathrm{mm}$ (mean $12.4(3.4) \mathrm{mm}$ ).
The choroidal melanomas $(n=66)$ demonstrated a height between 3.5 and $21 \mathrm{~mm}$ (mean $8.3(3.2) \mathrm{mm}$ ) and a base diameter between 4.4 and $22 \mathrm{~mm}$ (mean 12.8 (3.3) $\mathrm{mm}$ ).

REFLECTIVITY (FIG 1)

Metastases had a significantly higher reflectivity in A-scan than melanomas $(\mathrm{p}<0.001)$. The reflectivity in A-scan of the metastases ranged between $60 \%$ and $90 \%$ (mean $76 \%$ (SD $12 \%)$ ). The mean reflectivity of the melanomas was $22 \%$ ranging between $0 \%$ and $70 \%$ (SD $15 \%)$.

HEIGHT TO BASE RATIO (FIG 1)

For each tumour the height to base ratio was calculated, referring to the maximal prominence and the maximal base diameter of the tumour. Metastatic tumours had significantly lower height to base ratios that melanomas $(\mathrm{p}<0.001)$. The metastases demonstrated height to base ratios ranging between 0.08 and 0.31 (mean 0.18 (SD 0.08)), whereas the height to base ratios of the melanomas ranged between 0.23 and 0.95 (mean 0.6 (SD 0.16)).

SHAPE

Metastatic tumours had more frequently a polygonal surface in comparison with choroidal melanomas $(\mathrm{p}<0.001): 13$ metastases had a polygonal surface, three showed a domeshaped contour. No mushroom-shaped surface was observed among the metastases. Forty six of the 66 choroidal melanomas had a domeshaped surface, 14 a mushroom-shaped surface, and six had a polygonal surface.

CHOROIDAL EXCAVATION

Choroidal excavation was significantly more frequent in choroidal melanomas than in metastases $(p<0.001)$. Thirty eight melanomas demonstrated choroidal excavation, whereas no choroidal excavation was observed among the metastatic tumours. All melanomas that showed choroidal excavation had a maximal prominence of less than $8 \mathrm{~mm}$ (mean 5.8 (SD 0.8) $\mathrm{mm}$ ).

STATISTICAL ANALYSIS

A complete discrimination of the tumours concerning the diagnosis (choroidal metastasis $v$ choroidal melanoma) was achieved by the variable reflectivity or height to base ratio, using Fisher's exact test and $\chi^{2}$ test. With regard to this complete discrimination no multivariate statistical procedure was necessary.

\section{Discussion}

Recent studies have shown that the incidence of intraocular metastasis is much higher than it was postulated earlier. The incidence is approximately $8-10 \%$ with a clear preponderance of metastases to the posterior choroid. ${ }^{78}$

Typical clinical features have been described for both choroidal melanoma and metastasis of carcinoma, where the diagnosis is often supported by the presence of a history of systemic cancer. Nevertheless, the clinical appearance of choroidal melanoma and choroidal metastasis 
from carcinoma may be very similar and the diagnosis is quite difficult in these cases (especially in the absence of a history of systemic cancer).

Ultrasonography remains a very important method in the clinical evaluation of intraocular tumours. Differences of the ultrasonographic picture are based on the differences in the histoarchitecture of metastatic tumours and choroidal melanomas. A metastatic tumour from the breast presents histologically as solid epithelial nests or glandular structures, providing echo producing interfaces, resulting in high reflectivity and an irregular internal structure in ultrasonography. ${ }^{9}{ }^{10}$ Melanomas are densely cellular masses of low to moderate vascularisation with only a few necrotic areas, thus containing only few echo producing interfaces. As a result these tumours are of low to medium reflectivity with a regular internal structure. ${ }^{10-15}$ These findings were confirmed by our study; metastasis from breast carcinoma had a significant higher reflectivity than melanomas $(\mathrm{p}<0.001)$.

Metastatic tumours have a different growth pattern in the uvea than melanomas. Metastases appear to infiltrate and replace the normal choroidal architecture more diffusely than melanomas. ${ }^{1312}$ Melanomas are slowly growing tumours and they usually expand beneath an intact Bruch's membrane. They typically assume a nodular configuration and grow as fairly circumscribed thickening of the choroid. By further growth melanomas perforate Bruch's membrane and assume a mushroom-shaped configuration. ${ }^{11} 1214$ That different growth pattern of metastases and melanomas has been confirmed by the ultrasonographic finding in this study. There was a significant higher height to base ratio in melanomas (mean 0.6 (SD 0.16)) than in metastases (mean 0.18 (SD 0.08)). Coleman et al described a different height to base ratio for melanomas and metastases 20 years ago, however without providing numerical data. ${ }^{16}$ Perri et al described in 42 patients with choroidal metastases a flat infiltrating and irregular shape in B-scan ultrasonography, supporting this characteristic finding. ${ }^{9}$ This indicates that the height to base ratio in ultrasonography is of potentially clinical value for the discrimination of choroidal melanomas from metastasis of carcinomas.

Furthermore, the combined use of reflectivity and height to base ratio may constitute a highly significant variable for the discrimination of melanomas from metastases, as it was demonstrated by our study (Fig 1). This interesting finding has to be confirmed by further studies, including choroidal metastases from other carcinomas (for example, lung).

Choroidal excavation is a typical finding in choroidal melanomas, but it can also be produced by other choroidal lesions. ${ }^{12-14} 17$ The presence or absence of choroidal excavation in a choroidal lesion depends on the difference in reflectivity between the normal choroidal tissue and the abnormal tissue replacing the choroid. In our study choroidal excavation was significantly more frequent in choroidal melanomas than in metastases ( $p<0.001) ; 58 \%$ of melanomas demonstrated this phenomenon whereas no choroidal excavation was observed among the metastatic tumours. This supports the findings of other authors: Fuller et al described a choroidal excavation in $65 \%$ of melanomas and Coleman et al found choroidal excavation in $42 \%$ of melanomas. ${ }^{12}{ }^{13}$ Verbeek demonstrated a choroidal excavation in $70 \%$ of melanomas and approximately $20 \%$ of metastases. ${ }^{15} \mathrm{Al}-$ though he found in his analysis of echographic findings in 325 melanomas, 44 metastases, 19 haemangiomas and other intraocular tumours, a correct diagnosis determined by ultrasonography in $95 \%$ of cases, the differentiation of metastases from other tumours presented as the most difficult problem. ${ }^{15}$

In conclusion, this study demonstrates that the height to base ratio alone and the combined use of height to base ratio and reflectivity enable a highly significant discrimination between choroidal melanomas and metastases from the breast, thus probably constituting appropriate variables for clinical differentiation. Further studies are necessary to confirm this assumption.

1 Ferry AP, Font RL. Carcinoma metastatic to the eye and orbit. I A clinicopathologic study of 227 cases. Arch Ophthalmol 1974;92:276-86.

2 Shields JA. Metastatic tumors to the uvea and retina. In: Diagnosis and management of intraocular tumors. St Louis: Diagnosis and management

3 Stephens RF, Shields JA. Diagnosis and management of cancer metastatic to the uvea: a study of 70 cases. Ophthalcancer metastatic to the u
mology $1979 ; 86: 1336-49$.

4 Shields JA, Shakin EP, Shields CL. Metastatic malignant tumors. In: Gold DH, Weingeist TA, eds. The eye in systemic disease. Philadelphia: J B Lippincott 1990:299-303.

5 Shields JA, Shields CL. Metastatic tumors to the intraocular structures. In: Intraocular tumors. Philadelphia: Saunders, 1992: Ch 12, 207-38

6 Wetheimer R, Maier M, Hofmann H-M, Stanowski A, Weiss M. Karzinoidtumor der Lunge. Ophthalmologe 1993;90: $522-7$.

7 Mewis L, Young SE. Breast carcinoma metastatic to the choroid. Ophthalmology 982;89:147-51.

8 Nelson CC, Hertzberg BS, Klintworth GK. A histopathologic study of 716 unselected eyes in patients with cancer at
the time of death. Am 7 Ophthalmol 1983;95:788-93.

9 Perri P, Chiarelli M, Monari P, Ravalli L, Mazzeo V. Choroidal metastases. Echographic experiences from 42 patients. Acta Ophthalmol 1992;204:96-8.

10 Verbeek AM, Thijssen JM, Cuypers MHM, Brink H, Deutmann AF. Echographic classification of intraocular tumours. A 15-year retrospective analysis. Acta Ophthalmol 1994;72:416-22.

11 Ossoinig KC, Bigar F, Kaefring SL. Malignant melanoma of the choroid and ciliary body. Bibl Ophthalmol 1975;83: $141-54$

12 Coleman DJ, Abramson DH, Jack R. Ultrasonic diagnosis of tumors of the choroid. Arch Ophthalmol 1974;91:344-54.

13 Fuller DG, Snyder WB, Hutton WL, Vaiser A. Ultrasonographic features of choroidal malignant melanomas. Arch Ophthalmol 1979;97:1465-72.

14 Hodes BL. Ultrasonographic diagnosis of choroidal malignant melanoma. Surv Ophthalmol 1977;22:29-40.

15 Verbeek AM. Differential diagnosis of intraocular melanomas with ultrasonography. Ultrasound Med Biol 1985;II: mas with $163-70$.

16 Coleman DJ, Lizzi FL, Jack RL. Ultrasonography of the eye and the orbit. Philadelphia: Lea and Febiger, 1977:218.

17 v Gool CAM, Thijssen JM, Verbeek AM. B-mode echography of choroidal melanoma; echographic and histological aspects of choroidal excavation. Int Ophthalmol 1991;15: $327-34$. 